\title{
REPRESENTACIÓN CIENTÍFICA Y PERSPECTIVISMO REALISTA. HACIA UNA ARTICULACIÓN NATURALISTA
}

Scientific representation and realist perspectivism. Towards a naturalist articulation

\section{Cecília Pierola ${ }^{1}$}

\begin{abstract}
RESUMEN
Este artículo argumenta a favor del proyecto de una teoría perspectivista de la representación científica, y de la idea de que el perspectivismo realista puede beneficiarse con los aportes del campo de la representación científica y de la aceptación de una actitud naturalista. Se repasa la clasificación de las teorías de la representación en sustantivas y deflacionarias y se muestra que la adopción de la representación científica como unidad de análisis no implica un compromiso realista. Se presentan los principales rasgos del perspectivismo realista actual en filosofía de la ciencia, y se destacan los mayores desafíos que enfrenta. Asimismo, se muestra que la representación científica como unidad de análisis permite una mejor articulación de las tesis perspectivistas. Finalmente, se propone la adopción de criterios de aceptabilidad epistémica neutrales a toda perspectiva y de una actitud naturalista para sortear las dificultades del perspectivismo realista.
\end{abstract}

Palabras clave: Representación. Perspectivismo. Realismo. Naturalismo.

\begin{abstract}
This paper argues for the project of a perspectivist theory of scientific representation, and for the idea that realist perspectivism can benefit from the contributions of the field of scientific representation and from the acceptance of a naturalistic attitude. The classification of theories of representation into substantive and deflationary is reviewed and it is shown that the adoption of scientific representation as the unit of analysis does not imply a realist commitment. The main features of the current realist perspectivism in philosophy of science are presented, and the major challenges it faces are highlighted. It is also shown that scientific representation as a unit of analysis allows a better articulation of the perspectivist thesis. Finally, the adoption of perspective-neutral criteria of epistemic acceptability and a naturalistic attitude are proposed to overcome the difficulties of realist perspectivism.
\end{abstract}

Keywords: Representation. Perspectivism. Realism. Naturalism.

\footnotetext{
${ }^{1}$ Universidad de Buenos Aires.

E-mail: ceciliapierola@gmail.com. ORCID: 0000-0002-1383-4608.
} 


\section{Introducción}

Este artículo argumenta a favor del proyecto de una teoría perspectivista de la representación, y de la idea de que el perspectivismo realista puede beneficiarse con los aportes del campo de la representación científica y de la adopción de una actitud naturalista. En primer lugar se mostrará que adoptar la representación como unidad de análisis epistemológico es una decisión neutral respecto de la disputa entre realismo y antirrealismo científicos. En segundo lugar se presentarán los rasgos principales del perspectivismo actual, así como los desafíos más importantes que enfrenta. Por último, se propone que una actitud naturalista puede ofrecer una clave para la superación de esos desafíos. Vaya esta propuesta como homenaje a Ronald Giere, en el año de su fallecimiento.

\section{Teorías de la representación científica}

Durante las últimas décadas ha estado en el primer plano de los debates en filosofía de la ciencia de tradición analítica el problema de la representación científica, surgiendo así numerosas teorías que buscan dar cuenta de ella. Si tomamos como fecha de aparición de la filosofía de la ciencia en tanto disciplina autónoma el período de entreguerras (DIEZ CALZADA, 1997), pueden establecerse tres grandes concepciones epistemológicas: la llamada "concepción heredada", la historicista y la concepción semántica. En cada una de ellas prevaleció el estudio de diferentes unidades de análisis entendidas como entidades con ciertas características.

La concepción heredada tomó como unidad de análisis las teorías científicas entendidas como sistemas axiomáticos empíricamente interpretados. Es decir, los sistemas hipotético-deductivos que caracterizan la ciencia según esta tradición son sistemas axiomáticos que ameritan un análisis sintáctico, bajo el supuesto de que la estructura de la formulación lingüística de una teoría es la estructura de la teoría misma. Por ello, esta concepción hizo énfasis en las reglas de formación de expresiones y en las de inferencia de unas a partir de otras, así como en las reglas de correspondencia entre términos teóricos y otros observacionales, preteóricos (DIEZ CALZADA, 1997, p. 46). De este modo, la diferencia entre los conjuntos de axiomas de una y 
otra teoría implicaría una diferencia entre las teorías, resultado indeseado incluso por los teóricos de esta concepción. Así vista, la capacidad informativa de una teoría científica, es decir, lo que dice de la realidad, queda en un lugar secundario respecto de cómo informa, esto es, cómo dice lo que dice, porque el análisis no está centrado en la relación entre la teoría (o las teorías) y alguna porción del mundo (aun cuando el contenido empírico fuera un requisito demarcatorio), sino en las relaciones lógicas entre los enunciados y términos de una teoría, o las relaciones lógicas entre los conjuntos de enunciados de más de una teoría.

La vertiente historicista generó como unidades de análisis entidades que se caracterizaban de manera esencial por ser diacrónicas, es decir, por mutar en el tiempo histórico. Ejemplo de ello son los paradigmas y los programas de investigación científica (DIEZ CALZADA, 1989). Esta escuela ubicó la actividad de los agentes y sus contextos (epistémicos, cognitivos, sociales, según las distintas teorías desarrolladas por sus representantes) como un elemento esencial en el análisis de la ciencia. Se trata, así, de una visión influenciada por los estudios históricos sobre la actividad, y por tanto más emparentada con la práctica científica como tal. Esto introdujo el problema crucial de la carga teórica en el acceso al conocimiento. De este modo, las construcciones teóricas ya no son concebidas solo como el producto final del trabajo científico, sino como entidades que operan en la actividad científica. Se ejerce así un desplazamiento a segundo plano de la cuestión de la verdad.

La concepción semántica, por su parte, tomó como unidad de análisis las teorías entendidas como colecciones de modelos. Señala Suárez (2015) que si bien la teoría continuó siendo la unidad de análisis en estos epistemólogos, por la definición misma dada a esa entidad, la atención se desplazó a los modelos, su naturaleza y su papel en el conocimiento científico. Si bien hay quienes definen los modelos de una manera restringida a entidades matemáticas, durante las últimas dos décadas ha ganado adhesión una comprensión más amplia de la noción, que llega a incluir hasta entidades físicas.

En palabras de Brandon Boesch ([s.d.]) "La representación científica es la importante y útil relación que se mantiene entre fuentes científicas (por 
ejemplo modelos, teorías y modelos de datos) y sus targets $^{2}$ (por ejemplo, sistemas del mundo real y objetos teóricos)"

De este modo, la representación queda en el centro de las preocupaciones epistemológicas de las últimas dos décadas, como candidata a ser la relación a elucidar si se quiere comprender el vínculo entre los productos de la ciencia y aquello que se pretende conocer. El análisis de la representación permite ubicar en primer plano lo que las teorías dicen acerca de sus targets, y en un plano secundario las cuestiones enunciativas, que no desaparecen de la escena, y por tanto no hacer depender a las fuentes científicas de una sintaxis en particular. A su vez, cuando se considera a la representación como una actividad, este análisis posibilita tomar en consideración aspectos contextuales, sin deshacerse de la cuestión de la verdad, que según la teoría adoptada podrá predicarse de los enunciados de una teoría (que serán verdaderos o no respecto de modelos), o de las hipótesis teóricas sobre el ajuste entre los modelos y sus targets.

Asimismo, hay quienes discuten la fertilidad del análisis de la representación para comprender la relación entre los modelos y sus targets. Este rechazo se fundamenta en que la representación supondría una posición realista, pero la elección del foco de análisis debería ser al menos neutral respecto del debate realismo-antirrealismo, porque, según estos autores, buena parte del trabajo científico no se comprende desde posiciones realistas sino que sus producciones parecen funcionar como instrumentos. Es la postura de Healey (2010) y de Lombardi y otros (2016).

En el próximo apartado argumentaremos que, contra estas posiciones, la representación como unidad de análisis es neutral respecto del debate realismo-antirrealismo.

\footnotetext{
${ }^{2}$ Conservamos este término en su idioma original. En español podría ser traducido con los sustantivos "objetivo" o "blanco", pero ninguno consigue atrapar el significado específico del término en el contexto académico relevante. "Objetivo" es una palabra que, aun en su forma sustantiva, tiene en español resonancias filosóficas con las que el término original no se compromete. "Blanco" es un vocablo demasiado metafórico para traducir un término estrictamente técnico. El target es aquello pretendidamente representado por un vehículo representativo, común y confusamente nombrado también como "representación".
} 


\section{Teorías sustantivas y deflacionarias}

Sobre la base de las caracterizaciones que las teorías de la representación ofrecen acerca de la relación representacional, se ha extendido en la literatura una clasificación amplia entre teorías sustantivas y teorías deflacionarias.

Las teorías sustantivas son aquellas que (a) ofrecen condiciones necesarias y suficientes para definir una relación de representación, y (b) entre esas condiciones se cuenta la generalización de la relación más allá del caso particular, con lo cual no tiene relevancia alguna el contexto o los usuarios. Lo esencial de la representación en estas teorías se juega en la relación entre vehículo (o fuente) y target (BOESCH, [s.d.]; SUÁREZ, 2015a). Otra caracterización de estas teorías señala que ellas enfatizan el carácter informacional de la representación, sobre la base de una relación objetiva entre vehículo y target (CHAKRAVARTTY, 2010).

Mientras tanto, las teorías deflacionarias (a) se limitan a ofrecer condiciones suficientes, que permiten la representación, (b) entendida como el empleo de la misma por parte de usuarios, (c) en un contexto particular, indexado. Así, la relación diádica entre vehículo y target no es esencial en la representación, sino que esta depende de la práctica, y por tanto es una relación al menos triádica en su esencia misma (BOESCH, [s.d.]; SUÁREZ, 2015a). En las teorías deflacionarias, entonces, se enfatiza el carácter funcional de la representación para la actividad científica (CHAKRAVARTTY, 2010).

Quienes sostienen que tomar la representación como unidad de análisis epistemológico supone una posición realista, lo hacen por medio de la identificación de representar con describir y la contraposición de ello con fines pragmáticos de las producciones científicas.

En la estrategia de Healey (2010), sobre la base del caso de la física cuántica y en diálogo con el empirismo constructivo de van Fraassen, se sostiene que 
(...) los estados cuánticos no generan probabilidades que describan o representen nada: más bien, generan instrucciones sobre cómo uno debería formar sus creencias. Estas creencias no se refieren a probabilidades, ni tampoco a ninguna propiedad del sistema cuántico cuyo estado las genera. (HEALEY, 2010, p. 545)

Es decir, se contrapone representar con generar instrucciones para la creación de creencias y se reivindica al pragmatismo como un mejor marco de comprensión de los estados cuánticos que el que proporcionaría una visión representacionalista sin lugar para la intencionalidad.

Por otra parte, Lombardi et. al. (2016) suponen que representar implica describir un sistema que se equipara con una realidad objetiva e independiente, lo cual se contrapone de manera excluyente con las funciones instrumentales que pueda tener un modelo. El argumento de los autores apela a los modelos incompatibles, que no podrían describir una misma realidad (no contradictoria) y por tanto no podrían representar. Estas maneras de referirse a la representación parecen tener como referencia las teorías sustantivas únicamente. Quizás por ello, finalmente, estos autores señalan que en las tesis deflacionarias nada queda de la representación justamente porque se ha debilitado el compromiso realista que supondría esa unidad de análisis.

Sin embargo, no hay razones suficientes para sostener que toda teoría de la representación científica, por su naturaleza misma, es realista. Algunos argumentos pueden ser ofrecidos para ello.

En primer lugar, es un hecho reconocido que existen destacados filósofos que se refieren a la representación científica y no pueden ser caracterizados como realistas científicos en el sentido tradicional. Resalta en este universo el caso de van Fraassen (2008):

Para entender la ciencia, debemos abordarla desde muchas direcciones. Me centraré en un aspecto que considero fundamental para la empresa científica: la representación de los fenómenos empíricos, por medio de artefactos, tanto físicos como matemáticos. La posición de que el éxito a este respecto es el objetivo definitorio de las ciencias empíricas es un tema empirista del que no me apartaré. De hecho, este foco en la representación se ajusta muy bien al tema empirista. (VAN FRAASSEN, 2008, p. 1)

Es decir, para van Fraassen el tópico de la representación científica es consistente con las posiciones empiristas. Pero no solo eso; van Fraassen afirma sostener una visión de la ciencia en la 
(...) que el criterio básico de éxito es la adecuación empírica en lugar de la verdad general, y [en la] que la aceptación de una teoría científica tiene una dimensión pragmática (guiar la acción y la investigación), pero no necesita suponer más creencia que la de que la teoría es empíricamente adecuada. (VAN FRAASSEN, 2008, p. $3)$.

De manera que van Fraassen, al tiempo que destaca la centralidad de la representación para la empresa científica, se aleja del típico motivo realista de la verdad (presente en cualquier noción de descripción), poniendo en su lugar la adecuación empírica, y compatibiliza estas posiciones con un pragmatismo epistémico: "no hay representación excepto en el sentido de que algunas cosas son usadas, hechas o interpretadas para representar otras cosas de tal o cual manera" (VAN FRAASSEN, 2008, p. 23)

Por supuesto, podría argumentarse que la propuesta teórica de van Fraassen es objetable, crucialmente en lo que refiere a qué significa que un modelo teórico represente (por ejemplo, en Borge, 2015). Pero aún en estos casos no está probada la inconsistencia entre la noción de representación y una posición antirrealista (en el caso de van Fraassen, respecto de las entidades inobservables), excepto por la petición de principio que supone identificar cualquier referencia a la representación científica con una posición realista.

También podría señalarse que van Fraassen sí es un realista (“de sentido común") en lo que refiere a los fenómenos, de modo que la adecuación empírica como relación primordial entre vehículo y target es una relación con entidades objetivas independientes de las teorías. Pero van Fraassen extiende la práctica de representar más allá de los fenómenos, y la compatibilidad con el antirrealismo queda al descubierto en la consideración de la tergiversación, de la posibilidad de representación de entidades inexistentes, y de la representación como uso, que ya fue señalada. Como otra evidencia de esa compatibilidad, puede agregarse el fundamento de que exista la llamada "objeción de la pérdida de realidad".

Con respecto a la tergiversación, van Fraassen señala que la distorsión en una representación podría ser necesaria para su éxito, otorgando a algunas tergiversaciones la categoría de representación, después de todo (VAN FRAASSEN, 2008, p. 13). Para justificar esta idea, el autor distingue 
entre "representación de" (que apunta a un referente) y "representación como" ("así o así", a modo de predicación sobre el referente), indicando que mientras la similitud puede ser útil para ubicar al referente, no es necesaria para el éxito de los propósitos por los cuales se representa a ese referente “así y así". Es decir, una representación distorsiva de una entidad puede ser exitosa en un cierto contexto interpretativo. De este modo, van Fraassen se aleja del compromiso de literalidad y de creencia en la verdad al menos aproximada de los productos de la ciencia, compromisos típicos de cualquier realismo científico, y destaca el papel de los contextos interpretativos.

Más aún, para van Fraassen es posible representar entidades inexistentes o no experimentadas. Tras tomar como ejemplo el enunciado "este gráfico representa el crecimiento de una colonia de bacterias", el autor afirma que "el segundo relatum puede no ser real", y aun así el enunciado que establece la representación, ser verdadero:

La representación es intencional en el sentido de relacionarse con la intención epistémica, en el sentido de ser sobre algo, tal como lo son la referencia (por alguien) y la predicación (por alguien). Pero así como el pensamiento puede dirigirse en este sentido a lo que no está presente, no experimentado, no conocido o incluso inexistente, así también puede cualquier uso de algo para representar algo. Al usarlo así, el usuario otorga un rol, el rol de representar tal o cual cosa, así o así. (VAN FRAASSEN, 2008, p. 27)

Queda claro, entonces, que la clave de la representación está en el uso, desplazando del centro cualquier compromiso con la existencia de entidades independientes de nuestra mente, y por tanto alejándose más aun de cualquier realismo.

Como una evidencia final de la compatibilidad de la adopción de la representación como unidad de análisis y el antirealismo, instanciada en van Fraassen, puede tomarse la objeción de la pérdida de realidad, que justamente denuncia ese antirrealismo. Según van Fraassen, cuando se intenta verificar una afirmación de adecuación de un modelo teórico, se lo compara con un modelo de datos. Es decir, se confronta una representación con otra. Por ello los críticos sostienen que no está presente la realidad en esta relación. Van Fraassen responde con la conocida fórmula de la tautología pragmática, que establece que las afirmaciones "la teoría es adecuada a los fenómenos" y "la teoría es adecuada a los fenómenos tal como son representados por no- 
sotros" son la misma afirmación para nosotros (VAN FRAASSEN, 2008, p. 259). De modo que en esta respuesta, la relación entre vehículo y target de la representación sigue siendo una relación entre representaciones, y no con una realidad independiente.

En segundo lugar, las teorías deflacionarias introducen los factores pragmáticos como parte de la naturaleza misma de la representación. Así, la intencionalidad sostiene la representación y resuelve objeciones lógicas que pudiera enfrentar una teoría que implique caracterizar la representación como una relación reflexiva, simétrica y transitiva, como sucede con algunas teorías sustantivas. Es decir, la idea de que la representación científica juega un papel crucial en la empresa científica no necesariamente excluye la centralidad de los factores pragmáticos.

Por último, incluso en las teorías sustantivas de la representación científica no es necesario identificar el target con un mundo real objetivo, independiente o único. El target de una representación científica podría ser un modelo de datos, observaciones, fenómenos u otras entidades que no comprometan a quien toma como unidad de análisis la representación (ni a quien la usa) con ningún mundo existente independientemente. Incluso si subyaciera una noción de verdad por correspondencia, esto no implica necesariamente un compromiso realista. Una interpretación correspondentista de la relación de representación, o una noción de verdad por correspondencia requiere el compromiso con un estado de cosas, pero no que ese estado de cosas sea independiente de la mente (KIRKHAM, 1995, p. 133). La caracterización completa de lo que vuelve sustantiva una teoría de la representación es compatible con la opción de un target no real en el sentido usual para el realismo.

Las teorías de la representación no se pronuncian sobre el problema del realismo. A pesar de esto, es posible detectar implicancias realistas o antirrealistas en cada propuesta (LUCERO, 2020) así como evaluar en qué marco se ajusta mejor una teoría de la representación fructífera. En lo que sigue se presentará la posición contemporánea conocida como perspectivismo en el ámbito de la filosofía de la ciencia analítica, que aparece como corolario del debate realismo-antirrealismo en las últimas décadas. 


\section{Perspectivismo como realismo científico}

La disputa entre realismo y antirrealismo científicos de las últimas décadas parece haber llegado a un cierto "punto muerto", tal como señala Anjan Chakravartty (2017, cap. 7). En el contexto de ese ocaso gana relevancia una posición epistémica que intenta construirse entre los polos de aquella disputa. El perspectivismo, si bien no es una completa novedad de los últimos tiempos, se ha desarrollado ampliamente desde hace unos quince años, buscando componer un sentido realista de nuevo tipo.

En principio, el perspectivismo no pretende pronunciarse sobre aspectos metafísicos (aunque los supone) sino solo epistémicos, destacando la condición situada de nuestro conocimiento. El rango de posiciones perspectivistas que se pretenden realistas es amplio, desde algunas que podrían dejarnos más cerca del relativismo, como quizás sea el caso de las de Giere (2006), hasta otras que parecen llevarnos a reduccionismos teóricos indeseados, como tal vez sea el caso de Massimi (2017). No nos ocuparemos aquí de las diferencias en el interior de esta corriente, sino del planteo general común que ofrece para comprender el conocimiento científico. Para ello, describiremos los rasgos básicos del perspectivismo realista y sus más notorios desafíos.

En primer término, estos autores adoptan como punto de partida la práctica científica misma. Dado que se trata de una actividad humana, sostienen que las posibilidades de acceso al conocimiento por medio de ella están restringidas por las limitadas capacidades humanas. Esta actitud es llamada "humildad epistémica" por Massimi (2017; MASSIMI; MCCOY, 2020). De esta consideración por la práctica científica se deriva la atención puesta en los elementos de contexto como determinantes en la calificación del conocimiento, respecto de los cuales la producción científica es relativa y en virtud de los cuales está restringida.

Mientras que las anteriores apreciaciones son compartidas también por el pragmatismo y numerosas posiciones antirrealistas, el perspectivismo al que referimos aquí se reclama realista. De ahí que en segundo término debe comprometerse con afirmaciones básicas a todo realismo, de un modo suficiente para ser considerado tal cosa o al menos ser compatible con él. 
Esas afirmaciones básicas son las que enumera Psillos (1999, p. xvii y ss.) y a las que refieren tanto Massimi (2017, p. 170-1) como Teller (2020, p. 51 y ss.). Naturalmente, el compromiso con estas afirmaciones tendrá una declinación apropiada para hacerlo compatible con la consideración de la práctica científica y lo que de ella se deriva.

(R1) La primera tesis realista es la metafísica, que afirma la existencia de un mundo independiente de nuestras mentes, mundo que es el objeto del conocimiento científico. Para algunos perspectivistas esto involucra también el compromiso con clases naturales (MASSIMI, 2017).

(R2) La segunda es la tesis semántica, que sostiene que las afirmaciones de las teorías científicas deben ser tomadas literalmente, y por tanto que tienen valores veritativos condicionados por sus dominios pretendidos.

(R3) La tercera es la tesis epistémica, que declara que aceptar una teoría, tal como lo hacemos con las de la ciencia madura y predictivamente exitosa, implica la creencia de que ella es verdadera o aproximadamente verdadera. Es decir, que ellas constituyen conocimiento sobre el mundo.

El contenido de estas tres tesis es sostenido por el perspectivismo, que a su vez ofrece algunos señalamientos.

En primer lugar, Massimi (2017) propone (P1) distinguir entre objetividad y verdad. Mientras que no existe un punto de vista epistémicamente privilegiado por ser único y objetivo, y por tanto la investigación científica es de hecho pluralista, esto no hace que haya que concluir que son esas investigaciones o las perspectivas las que dan forma a los hechos ni, por tanto, que ellas hacen la verdad de las teorías. Aceptar un pluralismo epistémico contra el objetivismo no supone deflacionar la verdad de este modo.

En segundo lugar, Teller (2020) advierte que (P2) las tesis realistas no deberían ni necesitan involucrar realismo referencial alguno, dados los problemas que ello acarrea. Mientras que en la enunciación de esas tesis por parte de Psillos se remite explícitamente a la referencialidad de los términos, Teller sostiene que un compromiso demasiado rígido con ella inevitablemente falla. La razón ofrecida por Teller es que el mundo es demasiado complejo y nuestras limitaciones demasiado grandes como para que sea posible que nuestros términos, especialmente los teóricos, logren unirse a alguna característica que determine la extensión del mismo. La alternativa es el 
uso idealizado de los términos referenciales y la admisión de que enunciados de existencia sean "aproximadamente verdaderos" en el sentido de comprender que la referencia es una idealización exitosa para una amplia gama de aplicaciones. Probablemente sea lo que supone Psillos cuando afirma, en el marco de la tesis realista epistémica, que "las entidades postuladas por [las teorías científicas maduras y predictivamente exitosas], o, en cualquier caso, entidades muy similares a las postuladas, habitan el mundo" (PSILLOS, 1999, p. xvii. Subrayado nuestro).

En tercer lugar, Teller advierte que se requiere un examen crítico de la noción de "verdad aproximada". (P3) La verdad aproximada depende del contexto y del interés. Una afirmación falsa es "aproximadamente verdadera" en un contexto dado, con ciertos propósitos, en ciertos aspectos y grados, y no en otros. Y dado que una parte de las condiciones de verdad son, entonces, contextuales, la referencialidad entendida del modo anterior también lo es. Esto no elimina el papel jugado por los estados de cosas independientes de toda perspectiva, "que en última instancia hacen que nuestras afirmaciones científicas sea verdaderas o falsas. (...) Sin embargo, nuestra capacidad para conocer esos estados de cosas (y, por lo tanto, para atribuir un valor de verdad a las afirmaciones relevantes) depende inevitablemente de las perspectivas o el contexto de uso." (MASSIMI, 2017, p. 171).

Ahora bien, Teller declara: "'Las cosas son lo que son y del modo en que son, y no son de otra manera' es una verdad [truism, en el original] a la que suscribo completamente" (2020, p. 62). Si descartamos que se trate de una afirmación tautológica, puede suponerse que lo que se quiere decir es que lo que las cosas sean y el modo en que sean es independiente de las perspectivas, y que virtualmente puede postularse acerca de ellas un punto de vista exacto, aunque inaccesible para nosotros. En seguida la pregunta evidente es ¿cómo lo sabe? ¿es una cuestión de fe? ¿hay razones metafísicas? ¿se trata de una necesidad metodológica para la actividad científica? Complementariamente, Massimi afirma que "para que sepamos que (X), por ejemplo, es cierto, tiene que ser el caso, no solo de que (X) coincida con algún estado de cosas del mundo, sino también que satisfaga los estándares perspectivísticos relevantes de adecuación funcional en su contexto de uso." (2017, p. 172). Es decir, la verdad supone dos condiciones, ninguna sufi- 
ciente por sí misma, ambas necesarias: en última instancia, la correspondencia con el mundo independiente, pero también la satisfacción de los estándares relevantes en un contexto dado. Se corre aquí el riesgo de que destacar esa última condición equivalga a, como reza el dicho, "hacer de la necesidad, virtud". De la primera condición es simplemente imposible saber si se cumple o no, fuera de toda perspectiva, por el punto de partida mismo del perspectivismo que declara la humildad epistémica y el contextualismo. ¿En qué consiste, entonces, realmente esa condición? ¿Cómo podemos asegurar que se satisface aun de manera aproximada? Si esa garantía está dada por las perspectivas, de las que depende nuestra capacidad para conocer, ¿no colapsa esa condición en la segunda?, y además, ¿cómo puede evitarse que toda perspectiva sea considerada tan buena como cualquier otra?

Estas preguntas se dirigen al mayor desafío del realismo perspectivista. Se trata de un reto bicéfalo, consistente en articular una propuesta basada en la ciencia como actividad humana, que esquive tanto el escepticismo como el relativismo. En definitiva, el desafío es cómo sostener los compromisos realistas en este marco perspectivista, para lo cual se requiere eludir la clásica objeción de la pérdida de la realidad (GHINS, 2012; IRANZO, 2014; KAISER; ROSENHAGEN; SUHM, 2006; NGUYEN, 2016) o aquellas basadas en la existencia de modelos incompatibles (LOMBARDI; ACCORINTI; MARTÍNEZ, 2016; MORRISON, 2011).

\section{Una articulación naturalista para una teoría perspectivista de la representación científica}

A pesar de la amplia difusión de la taxonomía de las concepciones sobre la representación científica desarrollada en el primer apartado de este artículo, que las divide en sustantivas y deflacionarias, también se ha argumentado en favor de combinaciones entre esos dos grandes grupos (FRIGG; NGUYEN, 2020), su compatibilidad y hasta su necesidad mutua (CHAKRAVARTTY, 2010). El movimiento correlativo ha sido una hegemonía de las concepciones intencionales en este campo, cuya no contradicción y complementariedad entre sí también han sido destacada (BOESCH, 2019). Ya hemos señalado que las teorías de la representación no necesitan comprome- 
terse en principio con una posición respecto de la disputa entre realismo y antirrealismo, aunque es posible rastrear en ellas implicancias en ese sentido. Entre las concepciones dentro de ese campo que destacan el papel de la intencionalidad se encuentra un amplio espectro que va desde algunas decididamente antirrealistas, como la deriva reciente de la teoría inferencial (SUÁREZ, 2015b), hasta otras con aspectos más aparentemente comprometidos con las tesis realistas, como la teoría funcional basada en la informatividad (BOLINSKA, 2013). Un examen detallado de esta gama puede encontrarse en Lucero (2020).

Quizás Giere $(2004,2006,2010)$ sea el mejor representante de la introducción del perspectivismo en el campo de las teorías de la representación científica. Desde su punto de vista, debemos entender las teorías y leyes científicas como perspectivas que permiten construir modelos representativos, donde el foco está puesto en la actividad de hacerlo, es decir, en la representación como acto.

Nos interesa aquí señalar que, a su vez, la introducción del modo de analizar la práctica científica que toma como unidad de análisis la representación puede robustecer al perspectivismo como posición epistémica. Numerosos aspectos pueden ser indicados en este sentido. En esta oportunidad haremos énfasis en uno de ellos, que está especialmente relacionado con el principal desafío del perspectivismo, ya mencionado, y permite comprender el valor de una actitud epistémica naturalista.

La noción de representación científica que toma como vehículos objetos tales como modelos, pero también gráficos, esquemas, mapas y hasta maquetas, permite introducir relaciones entre vehículo y target que no requieren de la noción de verdad. Entre las respuesta posibles a la pregunta acerca de en virtud de qué un vehículo representa un target se destacan las elaboradas por las teorías sustantivas, como las que remiten a la coincidencia entre estructuras de uno y otro, o a la similitud entre ellos. Esta última relación es la que puso de relevancia Giere, incorporándola en una comprensión de la representación basada en agentes, es decir, no estrictamente sustantiva.

Luego de una extensa argumentación plasmada de modo exhaustivo en Giere (1999), el autor concluye que los objetos que mejor se ajustan a la 
concepción de la representación como actividad y a la práctica científica misma no son las teorías y las leyes, sino los modelos (GIERE, 2004, p. 744). Estos pueden ser construidos a partir de principios, a veces explícitamente formulados y que suelen ser llamados "leyes" de "teorías", en alguno de los múltiples sentidos en que se usan esos términos. De modo que a esos principios no corresponde tomarlos como afirmaciones acerca del mundo, que por tanto podrían ser verdaderas o falsas, sino como reglas o patrones para la construcción de modelos. Estos últimos sí pueden evaluarse en términos de su ajuste al mundo (estrictamente, a los modelos de datos) y por lo tanto generarse hipótesis acerca de ese ajuste que sí pueden ser verdaderas o falsas; los modelos no pueden serlo porque no son entidades lingüísticas. A aquellos principios Giere también se refiere como "perspectivas" acerca del mundo (1999, p. 94). De este modo, para Giere representar no es sinónimo de describir, ni esto se opone a aspectos pragmáticos sino que, antes bien, los principios efectivamente funcionan generando instrucciones para la construcción de modelos. De esta manera, los supuestos que sostenían el rechazo por parte de Healey de la representación científica como relación para comprender la ciencia quedan puestos es duda.

Los modelos también pueden ser construidos a partir de principios contradictorios entre sí (GIERE, 2010, p. 281), o a partir de ningún principio, sino de una combinación de otros modelos empíricos, modelos de datos y técnicas matemáticas, como por ejemplo los modelos de simulación. Más aún, los científicos pueden utilizar modelos incompatibles entre sí (GIERE, 2004, p. 749-50) para distintos propósitos, como lo ejemplifica la representación del agua como una colección de moléculas o como un fluido continuo, en diferentes usos, es decir, con diferentes fines.

Esa relación de "modelado representacional" queda mejor explicada, según Giere, como una relación de similitud. Si bien la similitud es una relación aparentemente diádica y reflexiva, mientras que el modelado es una actividad poliádica y asimétrica, no se presenta aquí un problema. Desde el punto de vista de Giere es justamente la intencionalidad del agente la que determina la similitud representacional entre dos objetos. Esto se cumple porque es el agente quien define cuáles son los aspectos relevantes en que son similares esos objetos, en qué grados (o qué grados de semejanza serían 
suficientes para que asignemos relación de similitud), y, crucialmente, cuál es el vehículo y cuál el target de la representación (es decir, la direccionalidad). Advirtamos que la posición de Giere es un tipo de pragmatismo que busca sostener algo del sustantivismo en teorías de la representación científica, es decir, mostrar que no por estar la representación mediada por el agente, cualquier cosa puede ser vehículo representativo de cualquier otra. Habría algo en el vehículo mismo que, sin ser relativo al o a los agentes, permite u obtura la relación representacional con un determinado target.

De esta manera, las características de la relación de similitud, que posibilitan (y requieren) especificar aspectos y grados, parecen permitir resolver de un modo más claro el problema de la verdad aproximada de las afirmaciones de existencia que discute Teller (2020). Los vehículos (por ejemplo, las entidades postuladas por la ciencia) que se usan para representar una entidad o colección de entidades de las que habitan el mundo (para seguir en la formulación a Psillos) se ajustan a ellas en ciertos aspectos y grados. Estos son epistémicamente suficientes cuando cumplen con los estándares de la perspectiva en que se inscriben los agentes y su uso de esas representaciones.

Enfrentar el problema de si toda perspectiva es igualmente válida requiere la postulación de algún criterio de evaluación externo y neutral respecto de todas ellas. Dado que estamos tratando con perspectivas científicas, esos criterios podrían ser el respeto por la evidencia y el compromiso con la búsqueda de conocimiento (CHAKRAVARTTY, 2017), que reconocen la intencionalidad epistémica (LUCERO, 2020) en la actividad científica. El respeto por la evidencia supone que los modelos construidos en el seno de una perspectiva estén informados o sean sensibles a la evidencia científica brindada por la experiencia y la observación. Sea la similitud o sea otra la relación adoptada para elucidar el vínculo entre un vehículo y su target, ella debe ser establecida por un proceso que tenga a la evidencia como fuente, tal que ella pueda autorizar o desautorizar un vehículo como representativo de un target (es decir, la vulnerabilidad empírica debería ser un valor compartido por toda perspectiva con pretensión epistémica). Para que el respeto por la evidencia sea efectivo como criterio externo y neutral a toda perspectiva es necesario que se compartan estándares mínimos sobre lo que cuenta como 
evidencia. Pero puede conservarse la pluralidad en el compromiso acerca de cuánto o qué es lo que esa evidencia permite inferir. La interpretación de la evidencia es una tarea informada por la perspectiva en que se esté trabajando. Complementariamente, el compromiso con la búsqueda de conocimiento implica la intencionalidad de ir más allá de lo ya conocido, y por tanto una cierta audacia epistémica característica de la práctica científica. Ello comporta la adopción de consideraciones no empíricas para inferir conocimiento a partir de la evidencia. La efectividad de este segundo criterio en tanto neutral y externo a toda perspectiva requiere que se compartan entre ellas ciertos estándares de métodos aceptables de análisis de la evidencia. Aquí también hay lugar para una pluralidad de perspectivas, por ejemplo según cuáles sean (además de la racionalidad lógica) las consideraciones no empíricas a tener en cuenta, qué valor de le otorgue al poder explicativo, y otras cuestiones de ese tipo.

No cualquier perspectiva se ajusta a la evidencia aceptada ni es funcional al objetivo de conocer, que orienta toda actividad científica, al menos parcialmente. Dado que de lo que se trata es de conservar los compromisos realistas, aquello que se busca conocer debe ser entendido como una entidad independiente de toda perspectiva, pero para cuyo acceso estamos limitados por las capacidades humanas y mediatizados por las perspectivas.

Ahora bien, la interfaz entre lo real y el conocimiento que podemos tener de ello no es misteriosa si aceptamos los desarrollos más avanzados de las ciencias cognitivas contemporáneas. Ellas caracterizan las determinaciones de las capacidades cognoscitivas humanas y muestran que la observación, por ejemplo, no es completamente dependiente del mundo lingüístico o cultural de quien percibe, sino que hay patrones de reconocimiento que se repiten ampliamente. Giere (1999, p. 100 y ss.) ofrece algunos valiosos ejemplos de esto, que podrían mostrar que cierto rango en la graduación de la similitud es compartida por sujetos que participan de diferentes contextos lingüísticos (1999, p. 104).

Si esto es así, es posible justificar la idea de algunos representantes del realismo perspectivista que afirma que podemos comprometernos con la existencia independiente de entidades y clases (MASSIMI, 2017, p. 174) $\sin$ apelar a actos de fe. Asimismo, es posible construir una vía de escape para el 
riesgo del escepticismo, dado que se puede apelar a evidencia parcialmente legitimada de manera neutral a toda perspectiva. Tenemos así un fundamento para creer que el mundo real efectivamente constriñe las representaciones aceptables dentro de cada perspectiva, tal que es posible conocer, parcialmente, si ellas se ajustan al mundo que "en última instancia" las confirma. Ahora cobra pleno sentido la distinción entre las dos condiciones de aceptabilidad de un realismo perspectivista: la similitud de la representación con algún estado de cosas del mundo, y la satisfacción por parte de esa representación de los estándares perspectivísticos relevantes de adecuación funcional en su contexto de uso.

\section{Conclusiones}

Tras haber repasado las características que las teorías sustantivas y las deflacionarias asignan a la representación científica, hemos argumentado que la adopción de ésta como unidad de análisis epistemológico no compromete con una posición realista, sino que es neutral respecto a la disputa realismo-antirrealismo. Volviendo la atención sobre esta disputa, presentamos la posición conocida como realismo perspectivista, que se propone construir un realismo no objetivista, en un momento de ocaso del debate. Destacamos el perspectivismo de Giere para abordar el problema de la representación científica como una actividad, y mostramos que la representación científica como unidad de análisis epistemológico permite una mejor articulación de las tesis perspectivistas. Por último, indicamos un camino posible para sortear las principales objeciones al realismo perspectivista en el campo de la representación científica. Este camino incluye la adopción, por un lado, de criterios de aceptación neutrales a toda perspectiva (el respeto por la evidencia y la búsqueda de conocimiento), y por otro lado, de una actitud naturalista que aborde el acceso humano al conocimiento del mundo beneficiándose de los desarrollos más recientes de las ciencias cognitivas. 


\section{Referencias}

BOESCH, B. Resolving and Understanding Differences Between AgentBased Accounts of Scientific Representation. Journal for General Philosophy of Science, v. 50, n. 2, p. 195-213, jun. 2019.

BOESCH, B. Scientific Representation. Internet Encyclopedia of Philosophy, [s.d.].

BOLINSKA, A. Epistemic representation, informativeness and the aim of faithful representation. Synthese, v. 190, n. 2, p. 219-234, jan. 2013.

BORGE, B. Modelos y representación en el Estructuralismo Empirista de Bas van Fraassen. Praxis Filosófica, Nueva Serie. v. 41, p. 27-42, dez. 2015.

CHAKRAVARTTY, A. Informational versus Functional Theories of Scientific Representation. Synthese, v. 172, n. 2, p. 197-213, 2010.

CHAKRAVARTTY, A. Scientific ontology: integrating naturalized metaphysics and voluntarist epistemology. Oxford, UK : New York, NY: Oxford University Press, 2017.

DIEZ CALZADA, J. A. La revuelta historicista en filosofía de la ciencia. Arbor, n. 526, p. 69-96, 1989.

DIEZ CALZADA, J. A. La concepción semántica de las teorías científicas. Éudoxa: Series Filosóficas, v. 8, p. 41-91, 1997.

FRIGG, R.; NGUYEN, J. Scientific Representation. The Stanford Encyclopedia of Philosophy, Spring 2020.

GHINS, M. Representation and the loss of reality objection. Epistemologia, v. 35, p. 47-58, 1 jan. 2012.

GIERE, R. N. Science without laws. Chicago: University of Chicago Press, 1999.

GIERE, R. N. How Models Are Used to Represent Reality. Philosophy of Science, v. 71, n. 5, p. 742-752, dez. 2004.

GIERE, R. N. Scientific Perspectivism. Chicago: University of Chicago Press, 2006.

GIERE, R. N. An agent-based conception of models and scientific representation. Synthese, v. 172, n. 2, p. 269-281, jan. 2010. 
HEALEY, R. Science without Representation. Analysis, v. 70, p. 536-547, 1 jul. 2010.

IRANZO, V. Models and Phenomena: Bas van Fraassen's Empiricist Structuralism. In: GONZALEZ, W. J. (Ed.). . Bas van Fraassen's Approach to Representation and Models in Science. Dordrecht: Springer Netherlands, 2014. p. 63-76.

KAISER, M. I.; ROSENHAGEN, R.; SUHM, C. Experience Becoming Fully Literate. Van Fraassen on the Verge of Constructivism. In: BERGHILDEBRANDT, A.; SUHM, C. (Eds.). . The Philosophy of Bas C. van Fraassen. [s.1.] ontos, 2006. p. 69-79.

KIRKHAM, R. L. Theories of truth: a critical introduction. Cambridge: MIT Press, 1995.

LOMBARDI, O.; ACCORINTI, H.; MARTÍNEZ, J. C. Modelos científicos: el problema de la representación. Scientiae Studia, v. 14, n. 1, p. 151-174, 2016.

LUCERO, S. El papel de la intencionalidad epistémica en las representaciones científicas. Compromisos realistas y anti-realistas. In: BORGE, B.; GENTILE, N. (Eds.). . La ciencia y el mundo inobservable: discusiones contemporáneas en torno al realismo científico. [s.1.] Eudeba, 2020.

MASSIMI, M. Perspectivism. In: SAATSI, J. (Ed.). . The Routledge Handbook of Scientific Realism. Routledge Handbooks in Philosophy. [s.1.] Routledge, 2017. p. 164-175.

MASSIMI, M.; MCCOY, C. (EDS.). Understanding Perspectivism. Scientific Challenges and Methodological Prospects. e-book ed. New York: Routledge, 2020.

MORRISON, M. One phenomenon, many models: Inconsistency and complementarity. Studies In History and Philosophy of Science Part A, v. 42, p. 342-351, 1 jun. 2011.

NGUYEN, J. On the Pragmatic Equivalence between Representing Data and Phenomena. Philosophy of Science, v. 83, n. 2, p. 171-191, 1 abr. 2016.

PSILLOS, S. Scientific Realism: How Science Tracks Truth. [s.1: s.n.].

SUÁREZ, M. Representation in Science. In: HUMPHREYS, P. (Ed.). . The Oxford Handbook of Philosophy of Science. Oxford handbooks. On line ed. New York: Oxford University Press, 2015a. p. 440-459.

SUÁREZ, M. Deflationary Representation, Inference, and Practice. Studies in History and Philosophy of Science, v. 49, p. 36-47, $2015 \mathrm{~b}$. 
Perspectiva Filosófica, vol. 48, n. 1, 2021

TELLER, P. What Is Perspectivism, and Does It Count as Realism? In: MASSIMI, M.; MCCOY, C. (Eds.). . Understanding Perspectivism. Scientific Challenges and Methodological Prospects. New York: Routledge, 2020. p. 49-64.

VAN FRAASSEN, B. Scientific Representation: Paradoxes of Perspective. New York: Oxford University Press, 2008. 